\title{
A REDSHIFT SURVEY OF IRAS GALAXIES
}

\author{
Michael A. Strauss and Marc Davis \\ Astronomy and Physics Departments \\ University of California, Berkeley \\ Berkeley, California, 94720
}

\begin{abstract}
We have completed a redshift survey of approximately 2200 IRAS galaxies, flux limited at $60 \mu$. The survey covers $76 \%$ of the sky and has a characteristic depth of $\approx 6000 \mathrm{~km} \mathrm{~s}^{-1}$, making it ideal for large scale structure studies requiring whole sky coverage. We have calculated the gravitational acceleration on us due to the inhomogeneous distribution of galaxies in the sample by summing the dipole acceleration in successive shells centered on us. The acceleration converges at $\approx 4000 \mathrm{~km} \mathrm{~s}^{-1}$ and we derive density estimates in the range $0.4<\Omega<0.9$. We discuss the various biases of the sample in detail: the paucity of elliptical galaxies, the problem of extended sources, and hysteresis, and suggest ways to accommodate them and thus decrease the uncertainty in $\Omega$. Finally, we discuss the use of the survey to make predictions for the peculiar velocity flowfield in space.
\end{abstract}

\section{INTRODUCTION AND SAMPLE SELECTION}

The IRAS database offers a unique opportunity to study the large-scale structure of the distribution of galaxies over large solid angle. It is estimated that the Point Source Catalog (hereafter PSC) contains some 20,000 galaxies (Soifer et al. 1987) that have been selected in a uniform way over the sky. Moreover, because galactic extinction is negligible in the IRAS bands, galaxies can be selected very close to the plane, at least until confusion makes the PSC unreliable. Meiksin and Davis (1986) and Yahil, Walker and Rowan-Robinson (1986) were the first to make galaxy catalogs from the PSC, and showed that the angular distribution of galaxies has a dipole moment that points very close to the peculiar velocity of the Local Group, as inferred from the dipole anisotropy of the Cosmic Microwave Background. The implication is that the IRAS galaxies at least approximately trace the matter that gives rise to our peculiar motion.

On large scales where linear perturbation theory applies, an object's peculiar velocity is directly proportional to its peculiar gravity where the constant of proportionality depends solely on $\Omega$, the cosmological density parameter. A redshift survey of an unbiased subsample of the IRAS galaxies offers the possibility of measuring $\Omega$ on the scale of the material giving rise to our peculiar velocity. The analysis of the peculiar gravity can determine the coherence length of expected peculiar velocities if the radius of convergence of our acceleration can be determined. Finally the survey can be used to construct the peculiar gravity field out to considerable distance. 
We have extracted a sample of 2407 objects from the PSC (first version) based on the Meiksin and Davis (1986) criteria: $f_{60} / f_{12}>3,|b|>10^{\circ}$, and $f_{60}>$ $1.936 \mathrm{Jy}$. The galactic latitude limit is imposed to avoid excessive contamination from galactic sources, to avoid problems of confusion at $60 \mu$, and to avoid problems of hysteresis after plane crossings (see, e.g., the IRAS Explanatory Supplement 1984). In addition, a few high-latitude star-forming regions, such as Orion and Ophiuchus, are excluded. The flux limit corresponds to the brightest quartile of the original Meiksin and Davis sample. The resulting sky coverage is 9.55 steradians, $76 \%$ of the sky.

In collaboration with John Huchra, Amos Yahil, and John Tonry, we have obtained optical spectra of the $\approx 60 \%$ of these objects that did not have redshifts in the literature, using telescopes at Cerro Tololo, Lick Observatory, and Mount Hopkins. Approximately $8 \%$ of the objects turn out not to be galaxies, but rather planetary nebulae, $\mathrm{T}$ Tauri stars, cirrus clumps, and other members of the galactic zoo.

We have included in our observing lists an additional 498 objects satisfying our color criterion, but with $f_{60}<1.936 \mathrm{Jy}$, and flagged as extended, weeks-confirmed, in bands 1, 2 or 3 . As we discuss below, these objects have point source fluxes that are underestimates of their true flux, and thus these objects may make it into our catalog. The IPAC people are currently ADDSCANing these sources for us, and we hope to have results by the end of the summer.

Finally, we are starting to observe galaxies in the unconfused regions of the excluded zones and in the region $5^{\circ}<|b|<10^{\circ}$ in an attempt to extend the survey to still greater sky coverage.

\section{THE SPACE DISTRIBUTION AND OUR PECULIAR GRAVITY}

In Figure 1, we present the distribution on the sky of the 2176 extragalactic objects in the survey as a function of redshift. Note how the level of anisotropy decreases markedly as a function of distance. All the well known clusters within $5000 \mathrm{~km}$ $\mathrm{s}^{-1}$ are identifiable in the distribution, but they are not as conspicuous as in an optically-selected catalog, because the IRAS galaxies are more loosely distributed than optical galaxies. A redshift-space correlation analysis of the IRAS galaxies shows that $\xi(s)$ is virtually identical to that of late type galaxies in the CfA survey for $s>2 h^{-1} \mathrm{Mpc}$. We find $\xi(s)=\left(s / s_{0}\right)^{-\gamma}$ with $s_{0}=5 h^{-1} \mathrm{Mpc}$ and $\gamma=1.8$ to be consistent with the data. On smaller scales the IRAS galaxies appear to be less clustered than typical spirals, but here the statistics are poor. These results are not surprising because the majority of IRAS galaxies are a dilute and apparently random subsample of all late type galaxies (Soifer et al. 1987).

Peebles (1980) gives the relation between peculiar velocity of an observer and the dipole moment of the matter distribution around him when linear growing modes predominate:

$$
\mathbf{V}=\frac{H_{0} \Omega^{0.6}}{4 \pi} \int d^{3} \mathbf{r} \delta(\mathbf{r}) \frac{\hat{\mathbf{r}}}{r^{2}}
$$

where $\delta(\mathbf{r})$ is the matter overdensity. In practice, we compute

$$
\mathrm{V} \Omega^{-0.6}=\frac{H_{0}}{4 \pi n_{1}} \sum_{i} \frac{1}{\phi\left(r_{i}\right)} \frac{\hat{\mathbf{r}}_{i}}{r_{i}^{2}}
$$



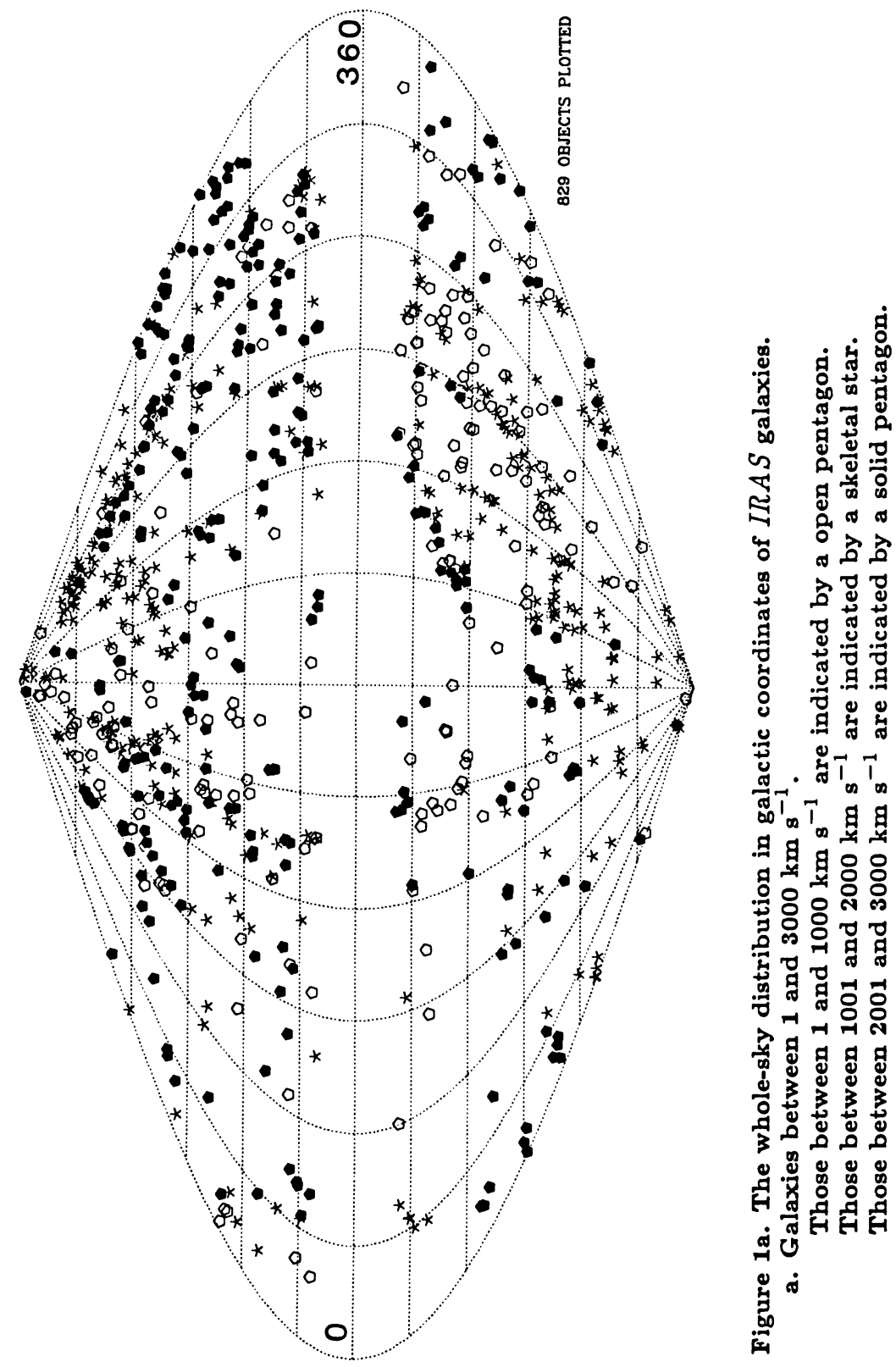


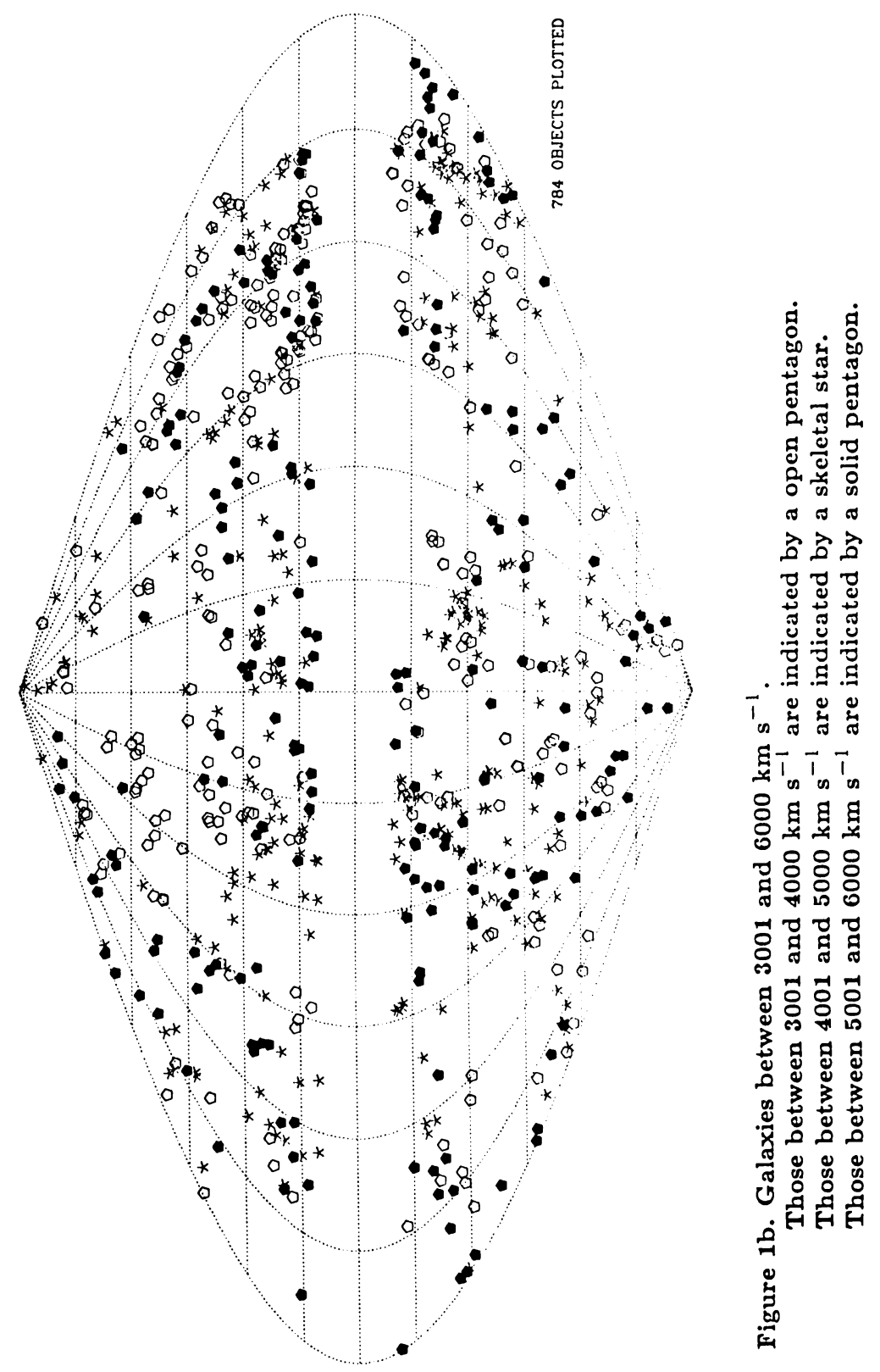


where the sum is over all galaxies sufficiently luminous to be observable to a redshift of $4000 \mathrm{~km} \mathrm{~s}^{-1}, n_{1}$ is the mean density of these galaxies, and $\phi(r)$ is the selection function, which is just the proportion of the luminosity function sampled at a given depth in the magnitude-limited sample (see Davis and Huchra 1982), and $\mathbf{r}$ is calculated assuming pure Hubble flow, after correcting redshifts for Virgocentric infall with a non-linear spherical model (Meiksin 1985). Our infall velocity was taken to be $250 \mathrm{~km} \mathrm{~s}^{-1}$, although our results are rather insensitive to this. The absolute magnitude limit imposed implies $\phi\left(v<4000 \mathrm{~km} \mathrm{~s}^{-1}\right)=1$ and we find $\phi(8000 \mathrm{~km}$ $\left.\mathrm{s}^{-1}\right) \approx 0.1$, which is as distant as we dare go in deriving results of cosmological significance. Finally, we must add a small correction to (2) to remove the dipole contribution of the excluded zones. It is important to note that our procedure is not a flux weighted sum; instead the sum is weighted by a function of the distance of each galaxy to correct for the fact that our sample is not volume limited. We assume implicitly that the IRAS galaxies roughly trace the mass and that their luminosity distribution is approximately independent of environment.

In Figure 2 we plot the three components of what we will call the "peculiar acceleration", $\mathrm{V} \Omega^{-0.6}$, as given by Eqn. (2), as a function of the limiting distance of the summation (solid pentagons). The $z$-axis points to the North Galactic Pole and the $x$-axis points to the Galactic Center. Our peculiar acceleration converges quite well at a distance of $4000 \mathrm{~km} \mathrm{~s}^{-1}$, with the bulk of the remaining fluctuations being consistent with a random walk induced by Poisson shot noise of the limited number of galaxies per shell.

The peculiar acceleration points in the direction $l=255^{\circ}, b=54^{\circ}$, only $26^{\circ}$ from the microwave dipole direction. Assuming $|\mathbf{V}|=600 \mathrm{~km} \mathrm{~s}^{-1}$, as implied from the observed microwave dipole anisotropy, the inferred value for $\Omega$ is 0.83 . However before we quote this as a conclusion, we must understand any and all sources of systematic error in the method. The first of these is the problem of extended sources. Any galaxy with an angular size greater than $\approx 100^{\prime \prime}$ at $60 \mu$ will be resolved by IRAS, and thus the $60 \mu$ flux listed in the PSC will be an underestimate of the true flux. In a first attempt to correct for this problem, we looked for matches in the SES Catalog of all sources flagged in the PSC I as extended in one of the first three bands. Unfortunately, only 1/3 of these sources had matches. As an alternative, we are having the IPAC people ADDSCAN each of these 900-odd sources. Until we have results from them, we will use the simple kludge of multiplying the Point Source flux of each extended source within a redshift of $5000 \mathrm{~km} \mathrm{~s}^{-1}$ by a factor two, a number based on our admittedly limited experience with ADDSCANing.

There are additional complications with the extended sources. There are certainly sources in our sample that will violate our color criterion once accurate fluxes are known for them: Similarly there must be numerous sources not in our sample which belong, rejected only because the inaccurate PSC fluxes do not satisfy the color criterion. Finally, a large number of the sources flagged as extended turn out to be quite distant galaxies $\left(v>5000 \mathrm{~km} \mathrm{~s}^{-1}\right)$, with angular size $\ll 100^{\prime \prime}$. These are extended because they are viewed through a foreground of Galactic cirrus, because they are in a group of infrared-bright galaxies, or because of some glitch in the IRAS processing. In any case, it is not yet clear to us how to treat these objects. The open pentagons in Figure 2 show the peculiar acceleration with the additional factor of two in flux for the extended sources. The curves have essentially the same shape as before, but the more numerous foreground galaxies increase the peculiar acceleration, resulting in a reduction of the inferred $\Omega$ to 0.67 . The direction of the 


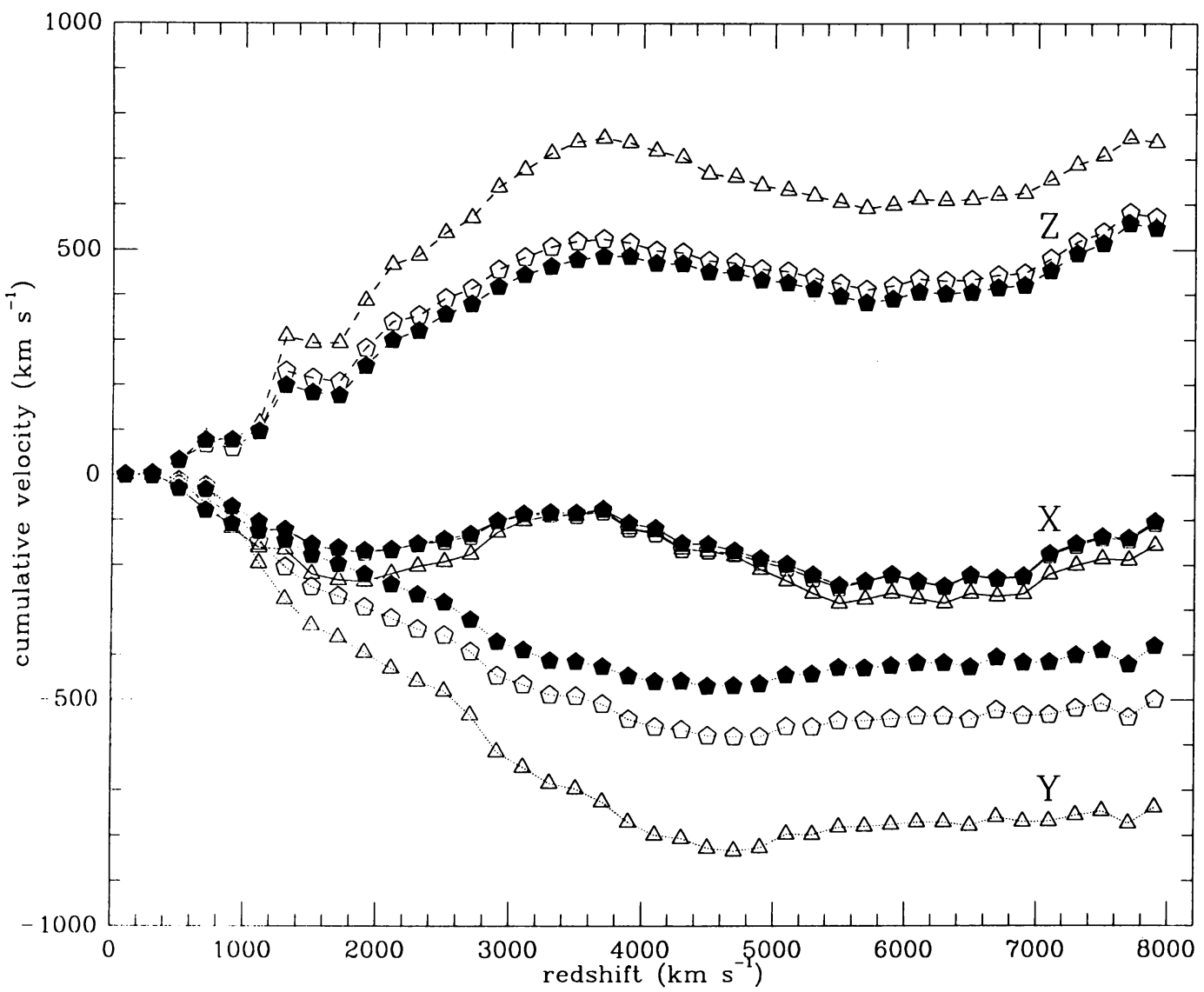

Figure 2. The three components of the cumulative peculiar gravity. The $\mathrm{X}$ component is the solid curve, $\mathrm{Y}$ is the dotted curve, and $Z$ is the dashed curve. The solid pentagons use the PSC flux. The open pentagons double the apparent flux for objects flagged as extended. The open triangle curves also double count those objects with the most neighbors. Note that the curves have very similar shapes, but different amplitudes.

calculated peculiar acceleration is now $20^{\circ}$ from the microwave vector and the convergence radius is approximately the same. Clearly, it is very important to properly account for extended sources!

Another bias in our sample is the fact that very few early type galaxies are included. The IRAS sample definitely gives low weight to cluster centers. Clusters such as Coma are completely invisible in the IRAS distribution, and the maps of Figure 1 show weaker concentration toward cluster centers than in equivalent optically selected samples. We have addressed this problem in two ways. First we considered an analysis of the peculiar gravity only in the 1.83 steradians of sky covered by the original CfA survey. The comparison of the z-component of the optical to IRAS "gravity" in this region of sky is shown in Figure 3. The two are remarkably similar, suggesting that the IRAS galaxies trace the large-scale 


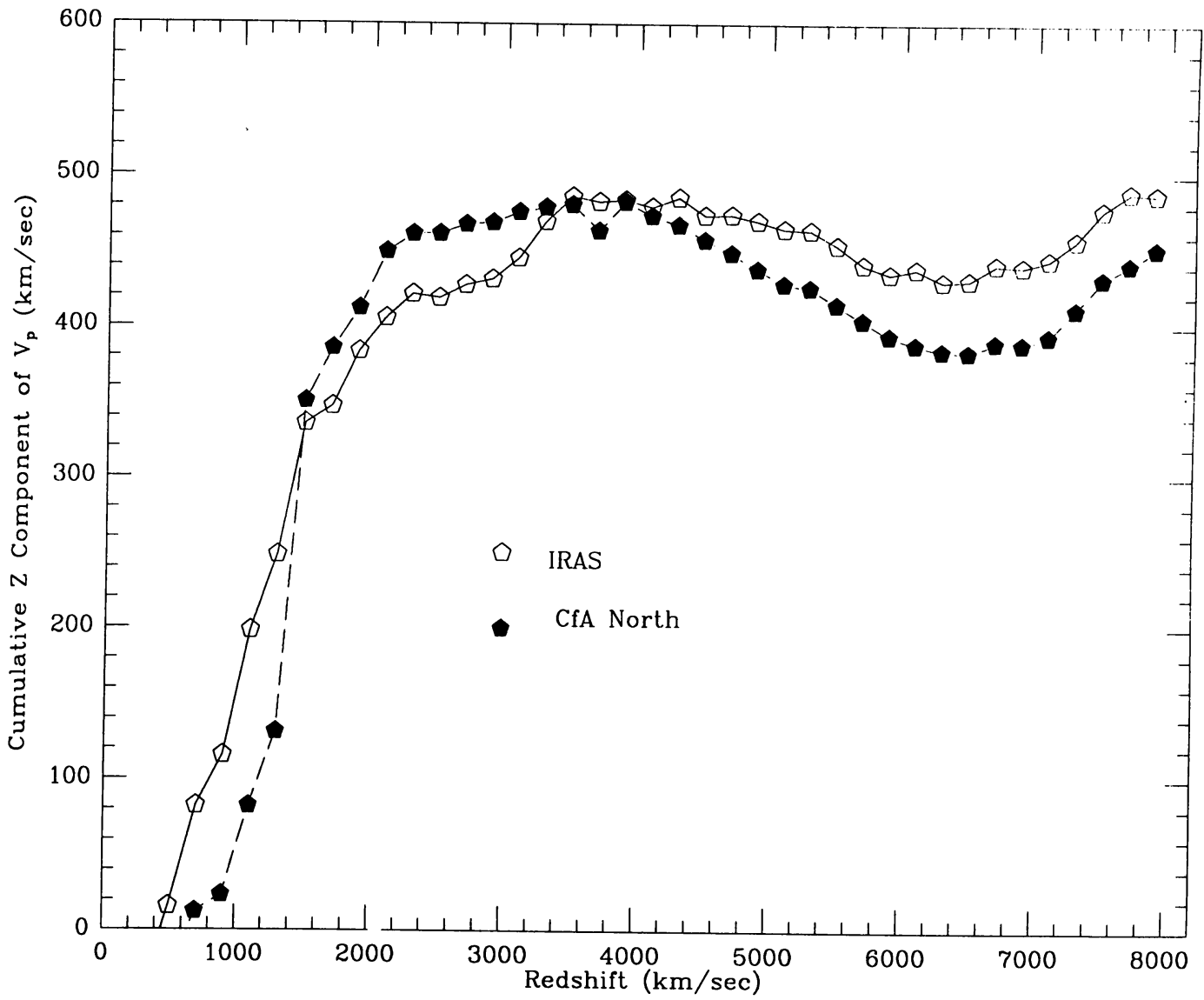

Figure 3. A comparison of the $z$-component of the peculiar gravity of the CfA survey (solid symbols) and the IRAS survey restricted to the same area of the sky (open symbols).

distribution of optically-selected galaxies very well, although the agreement is not perfect.

A test of the robustness of our results is to add the missing cluster cores by hand, as it were. Early type galaxies comprise typically $20 \%$ of the objects in optically selected magnitude-limited samples and are more clustered than late types. Therefore we simply double-counted the $25 \%$ of the IRAS galaxies that had the most companions within $7 \mathrm{~h}^{-1} \mathrm{Mpc}$ ( 5 or more companions). This again results in a larger peculiar gravity (open triangles in Fig. 2) and reduces $\Omega$ to 0.39 . The convergence radius remains nearly unchanged, but the convergence is more pronounced. The gravity vector moves to $l=258^{\circ}, b=44^{\circ}$, only $16^{\circ}$ from the microwave vector. This shows that indeed our results are sensitive to the addition of early-type galaxies. We are currently investigating other ways to quantify this systematic effect. 


\section{THE PECULIAR VELOCITY FLOW FIELD}

The completeness of sky coverage of the IRAS redshift survey allows us to extend the computation of the peculiar acceleration to observers at other points in space. For instance, we could put an imaginary observer at the center of the Virgo Cluster, and ask what her expected peculiar velocity is, due to the IRAS galaxies, by carrying out the sum in Eqn. (2). We have carried out this analysis for a grid of observers distributed over the sky, and have made maps of the expected peculiar velocity field. As above, we doubled the fluxes of sources flagged as extended. We added 424 random sources in the excluded zones, using the same selection function, to generate a catalog of sources with $100 \%$ sky coverage. Thus we are assuming that there are no large over- or under-densities lurking behind the Galactic Plane or Ophiuchus. The analysis was applied on a grid of points in latitude and longitude, on spherical shells centered on us. Figures $4 a$ and $4 b$ show the results, on shells at 2000 and $4000 \mathrm{~km} \mathrm{~s}^{-1}$, respectively. Beware of the Mercator projection of these plots; they can be misleading!

Figure $4 a$ is on the far side of Virgo, and thus we see strong infall with $V_{r}$ negative, centered at $l=290^{\circ}, b=+70^{\circ}$. We also see the effect of the Centaurus and Hydra clusters at $3000 \mathrm{~km} \mathrm{~s}^{-1}$ in the outflow with large shear centered at $l=300^{\circ}, b=+30^{\circ}$.

Figure $4 b$ is a slice just in front of Perseus, and the flow field is very strong there. The radial field in this region is dominated by the backwards pull of Hydra and Centaurus. The transverse fields in this region are rather jumbled, however; perhaps this is telling us something.

\section{THE WORK THAT LIES AHEAD}

Clearly, we are far from finished with this project. We have described above our first crude attempts to deal with two of the more blatant systematic effects of our sample, namely the problems of extended sources and of the paucity of early-type galaxies. The problem of hysteresis has not yet been addressed. We have recently discovered a strong anti-correlation between the Point Source Correlation Coefficient (CC) (IRAS Explanatory Supplement 1984) and the ratio of ADDSCANed to PSC flux; there is a large number of point sources with poor CC's, but not flagged as extended. Should all of these be ADDSCANed as well? We should also remake our sample based on the new version of the PSC; undoubtedly the galaxy list will change slightly.

The relatively short radius of convergence of the peculiar gravity implies that we do not expect large amplitude bulk flows with coherence length larger than this. The apparent disagreement of our peculiar gravity results with the velocity field measurements (Rubin, 1987; Collins et al. 1986; Dressler et al. 1987; Lynden-Bell et al. 1987) is very intriguing and must be investigated further. We have assumed pure Hubble flow throughout this discussion, while our results in Figures 4 clearly show this to be an inadequate assumption. Perhaps we can use these results to correct our observed redshifts for peculiar motions, and iterate. Over the next year we hope to reconcile the reported large scale velocity fields to the IRAS gravity field. 


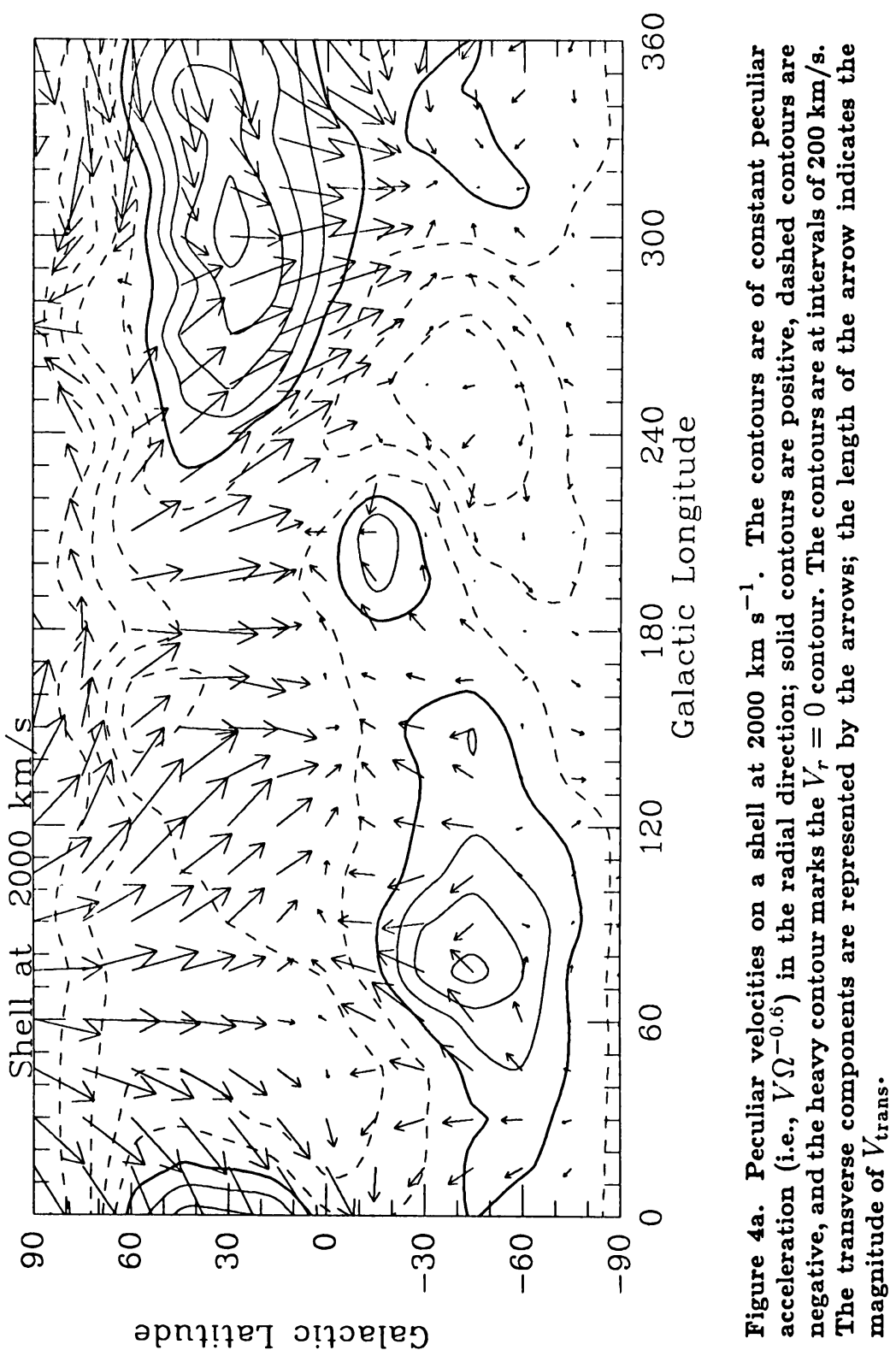




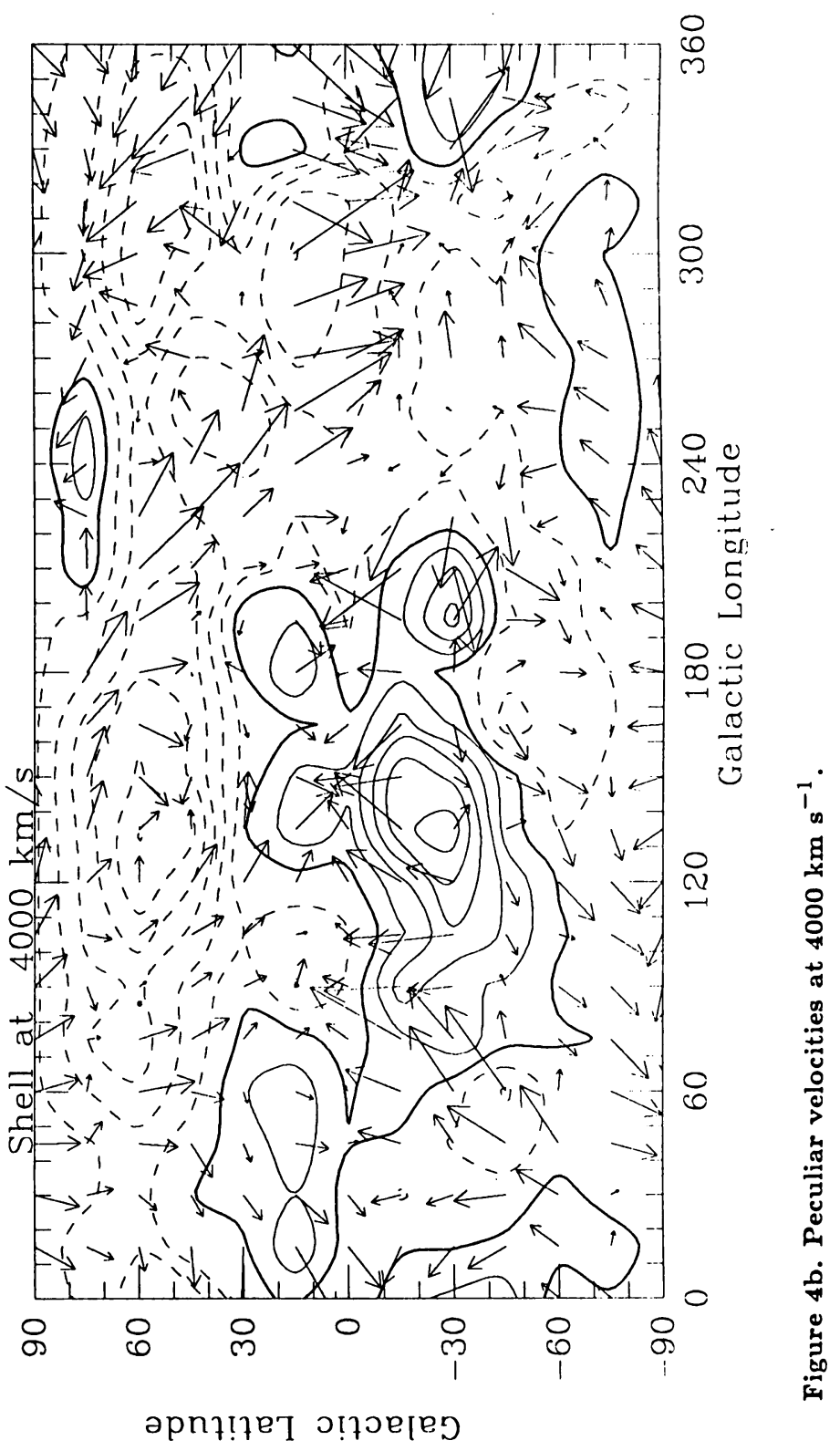


Current models of the nature of the dark matter pervading the Universe give definite predictions for the nature of the flowfield on different scales. The convergence radius inferred for our data is consistent with the currently popular Cold Dark Matter model, for instance.

Finally, we point out that we have perhaps the world's largest collection of spectroscopic data on the IRAS galaxies. We have just begun to study their emission-line properties, and hope to do detailed comparison with optically-selected samples of galaxies. A great deal of work lies ahead in digesting the mountains of data we have. The known systematic errors in the catalog appear to be treatable, and in a few month's time we hope to reduce the uncertainty in $\Omega$ and to better understand the limitations of the data base.

We thank our collaborators for allowing us to use as-yet unpublished data. Thanks to Avery Meiksin, for encouragement and constructive criticism at all stages of the project. This work is supported by the NSF and NASA/IPAC. MAS acknowledges the support of an NSF Graduate Fellowship.

\section{REFERENCES}

Collins, C. A., Joseph, R. D., and Robertson, N. A. 1986, Nature 320, 506.

Davis, M., and Huchra, J. 1982, Ap. J. 254, 437.

Dressler, A., Faber, S. M., Burstein, D., Davies, R. L., Lynden-Bell, D., Terlevich, R. J., and Wegner, G. 1987, Ap. J. (Letters) 313, L37.

IRAS Catalogs and Atlases, Explanatory Supplement 1985, edited by C. A. Beichman, G. Neugebauer, H. J. Habing, P. E. Clegg, and T. J. Chester ( Washis D. C.: U.S. Government Printing Office).

Lynden-Bell, D., Faber, S. M., Burstein, D., Davies, R. L. Dressler, A. J., Terlevich, R. J., and Wegner, G., 1987, Ap. J.

Meiksin, A. 1985, Private Communication.

Meiksin, A., and Davis, M. 1986, A. J. 91, 191.

Peebles, P. J. E. 1980, The Large-Scale Structure of the Universe (Princeton: Princeton University Press), §14.

Rubin, V. 1987, in "Large Scale Structure in the Universe", IAU 130.

Soifer, B. T., Houck, J. R., and Neugebauer, G. 1987, Ann. Rev. Astron. Astrophys., in press.

Yahil, A., Walker, D., and Rowan-Robinson, M. 1986, Ap. J. (Letters) 301, L1. 